\title{
MODELO MATEMÁTICO DAS FORÇAS DE NÃO-ARRASTE NO ESCOAMENTO BIFÁSICO DO DESGASEIFICADOR RH
}

Leonardo Neves

Roberto Parreiras Tavares ${ }^{2}$

\section{Resumo}

No presente trabalho foi feito um estudo do escoamento bifásico do desgaseificador RH em modelos físicos. A modelagem matemática do foi feita utilizando a abordagem de dois fluidos Euleriana-Euleriana. Foi feito um estudo da influência das diferentes forças de não-arraste, tais como: força de dispersão turbulenta, força de lubrificação da parede e força de sustentação. No estudo foi empregado o modelamento matemático na determinação da taxa de circulação no modelo físico do desgaseificador RH da USIMINAS na escala de I:5. Para a validação dos resultados do modelamento matemático, ensaios foram feitos utilizando estes modelos físicos empregando técnicas para obtenção de tempo de homogeneização de soluções salinas. O acompanhamento do escoamento foi feito através de filmagens utilizando câmera comum e câmera de alta velocidade. Foi determinado que as forças de não-arraste influenciam no escoamento bifásico ar/água sendo a combinação das forças de dispersão turbulenta, lubrificação das paredes e sustentação que possuíram os melhores resultados, ao serem comparados com os resultados experimentais.

Palavras-chave: Desgaseificador RH; Escoamento bifásico; Força de arraste; Forças de não-arraste; Taxa de circulação.

\section{MATHEMATICAL MODEL OF THE NON-DRAG FORCES IN TWO-PHASES FLOW OF RH DEGASSER}

\begin{abstract}
In the present work a biphasic flow study of the RH degasser was carried out in physical models. The mathematical modeling was made using the Eulerian approach. The influence of different non-drag forces was studied, such as: turbulent dispersion force, wall lubrication force and lift force. In the study, the mathematical modeling was used to determine the circulation rate in the physical model of the RH degasser of USIMINAS in the I:5 scale. For the validation of the mathematical model, tests were made in the physical model using techniques to obtain homogenization time of salt solutions. The flow monitoring was done by using ordinary camera and high-speed camera. It was determined that the non-drag forces have influence on the two-phase flow (air/water) and the combined forces of turbulent dispersion, wall lubrication force and lift force lead to best results, when compared with the experimental results. Non-drag forces were determined to influence the air / water two-phase flow, the combination of turbulent dispersion, wall lubrication and lifting forces having the best results compared to the experimental results.
\end{abstract}

Keywords: RH Degasser; Two-phase flow; Drag force; Non-drag forces; Circulation flow rate.

\section{INTRODUÇÃO}

A evolução da modelagem matemática durante os anos ocorreu de acordo com a evolução dos métodos de discretização, das metodologias matemáticas, da capacidade de processamento dos computadores empregados e de considerações feitas para formulação dos modelos. Vários autores utilizaram modelagem matemática em conjunto com o modelo físico ou com testes industriais para estudar a taxa de circulação e o comportamento do fluxo do aço líquido no interior do desgaseificador RH ("Ruhrstahl Heraeus") variando seus parâmetros dos quais se destacam Li e Tsukihashi [I], Kishan e Dash [2], Geng et al. [3,4], e Chen e He [5]. Li e Tsukihashi [I] propuseram um modelo matemático para o escoamento do aço no processo $\mathrm{RH}$ quando se aplica um campo eletromagnético rotativo na perna de subida

'Centro Federal de Educação Tecnológica de Minas Gerais - CEFET-MG, Belo Horizonte, MG, Brasil.

${ }^{2}$ Universidade Federal de Minas Gerais - UFMG, Belo Horizonte, MG, Brasil. E-mail: rtavares@demet.ufmg.br

2176-1523 (C) 2017 Associação Brasileira de Metalurgia, Materiais e Mineração. Publicado pela ABM. Este é um artigo de acesso aberto distribuído sob os termos da licença Creative Commons CC BY-NC-ND (Attribution-NonCommercial-NoDerivs) - https:// creativecommons.org/licenses/by-nc-nd/4.0\%. 
do $\mathrm{RH}$. Em um trabalho mais recente, Kishan e Dash [2] procuraram caracterizar o escoamento bifásico no interior de três equipamentos distintos em relação à distribuição do número de pernas utilizando a equação de movimento das bolhas composta por diferentes forças atuando na fase dispersa. Geng et al. [3,4] aplicaram em um modelo matemático do escoamento do aço líquido/argônio gasoso no desgaseificador $\mathrm{RH}$ utilizando forças interfaciais agindo na bolhas de argônio de acordo com o cálculo proposto por $\mathrm{Li}$ e Tsukihashi [I]. Chen e Shengping [5] propuseram a injeção no fundo do desgaseificador $\mathrm{RH}$ com o intuito de melhoria do fluxo e consequentemente na produção. Sabe-se que a força de arraste das partículas introduzidas na equação de conservação de quantidade de movimento é utilizada para simulação do escoamento multifásicos. No entanto as forças de não-arraste podem ser negligenciadas. As forças de não-arraste vêm sendo incorporadas por vários autores nos últimos anos em colunas de bolhas [6-8], com o principal objetivo de melhor representar os sistemas que possuem 0 escoamento multifásico. No presente trabalho foi feito um estudo da influência das diferentes forças de não-arraste no escoamento bifásico ar/água no modelo físico do desgaseificador $\mathrm{RH}$ aplicando diferentes forças interfaciais de não-arraste, tais como: força de dispersão turbulenta, força de lubrificação da parede e força de sustentação. Foram identificadas as combinações de forças que permitem a obtenção de previsões dentro dos limites dos resultados experimentais de taxa de circulação. Tal técnica utiliza traçadores para encontrar a taxa de circulação no aço líquido de obtenção de taxa de circulação, este método foi utilizado pelos seguintes autores Silva et al. [9] e Almeida et al. [10]. Para a validação das simulações matemáticas foram obtidos dados experimentais de taxa de circulação no modelo físico do desgaseificador $\mathrm{RH}$ da USIMINAS na escala de I:5. Neste trabalho procurou-se utilizar as forças de não-arraste com o principal objetivo de obter uma maior dispersão das bolhas, maior penetração e obter previsões de taxa de circulação que se aproximem dos resultados experimentais.

\section{METODOLOGIA}

Foi desenvolvido um modelo matemático do escoamento tridimensional bifásico água/ar aplicada ao modelo físico do desgaseificador $\mathrm{RH}$ construído na escala de $1: 5$ do desgaseificador $\mathrm{RH}$ industrial. As características do modelo físico do desgaseificador $\mathrm{RH}$ e as condições de injeção de gás são apresentadas na Figura I. O número e o diâmetro dos bicos foram utilizados de acordo com as condições encontradas na indústria. As propriedades das substâncias utilizadas são apresentadas na Tabela I.

As similaridades dinâmica e cinemática foram estabelecidas em função dos números adimensionais de Froude e de Froude modificado. Utilizando a similaridade desses grupos adimensionais no modelo físico com o equipamento industrial, pode-se encontrar a relação da vazão de gás injetado e dos diâmetros dos bicos de injeção.

As forças de arraste e não-arraste foram introduzidas no termo fonte da equação de conservação de quantidade de movimento da abordagem Euleriana. As forças de não-arraste consideradas neste trabalho foram as seguintes: força de sustentação, força de lubrificação da parede e força de dispersão turbulenta. Estas forças de não-arraste foram consideradas separadamente e também combinadas com - objetivo de ajustar os resultados de taxa de circulação com os experimentos. As faixas de valores dos coeficientes adimensionais utilizadas nas simulações propostas neste trabalho foram variados e combinados em patamares pré-definidos. $O$ coeficiente de força de sustentação, $C_{s}$, variou entre, 01 e I, 0 ; Os coeficientes da força de lubrificação das paredes, $C_{\text {I }}$ e $C_{2}$, variaram entre $-0,010$ e $-0,030$ e entre 0,050 e 0, 100 , respectivamente; $E$, o coeficiente da força de dispersão turbulenta, $C_{D T}$, variou entre $0, I$ e I, 0 . O coeficiente da força de sustentação adimensional é padronizado entre os valores de $0,0 \mathrm{I}$ a 0,5 para fluxos viscosos. Os coeficientes $C_{1}$ e $C_{2}$ da força de lubrificação das paredes é padronizado nos valores respectivos de $-0,01$ e 0,05 . Apesar de não existir um valor universalmente válido para o coeficiente da força de dispersão turbulenta, valores entre $0, \mathrm{I}$ a 0,5 têm sido utilizados com sucesso para o regime de borbulhamento com o diâmetro de bolhas na ordem de milímetros [II].

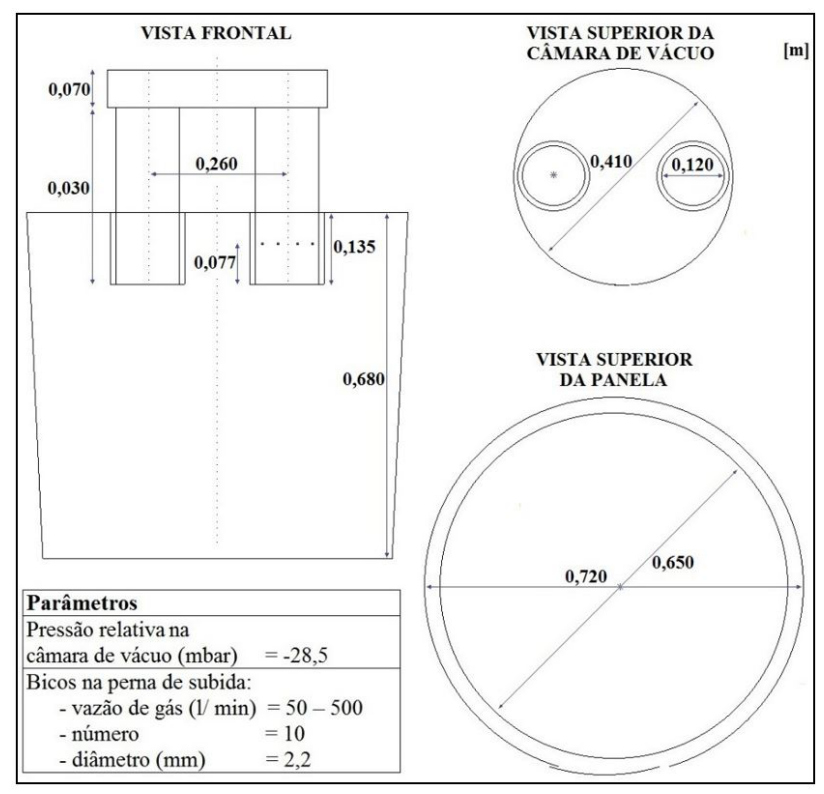

Figura I. Desenho esquemático do desgaseificador $\mathrm{RH}$.

Tabela I. Propriedades das substâncias utilizadas no modelamento matemático do escoamento no modelo físico

\begin{tabular}{lccc}
\hline & $\begin{array}{c}\text { Densidade } \\
\left(\mathbf{k g} / \mathbf{m}^{3}\right)\end{array}$ & $\begin{array}{c}\text { Viscosidade } \\
\text { Dinâmica }(\mathbf{k g} / \mathbf{m . s})\end{array}$ & $\begin{array}{c}\text { Calor Específico } \\
(\mathbf{J} / \mathbf{k g} . \mathbf{K})\end{array}$ \\
\hline $\mathrm{Ar}$ & Gás ideal & $1,831 \times 10^{-5}$ & 1004,40 \\
Água & 997 & $8,9 \times 10^{-4}$ & 4181,70 \\
\hline
\end{tabular}


O modelo de arraste entre as fases ar/água adotado o modelo de Grace et al. [12] com coeficiente igual a dois (2). Para este modelo foi utilizado a tensão interfacial de $0,08 \mathrm{~N} / \mathrm{m}$. Foi utilizado o diâmetro das partículas da fase dispersa igual a três milímetros [I]. Posteriormente, este valor foi substituídos por valores obtidos em resultados experimentais.

As equações de conservação foram discretizadas segundo o esquema "Upwind". Foram utilizados fatores de relaxação para viabilizar a convergência dos resultados através do controle de escala de tempo para o estado estacionário e transiente. Para a solução das equações, obtenção e análise dos resultados foi utilizado o software comercial de fluidodinâmica computacional da ANSYS [13]. Na solução das equações de conservação relativas ao escoamento de fluidos foi estipulado como critério de convergência RMS ("Root Mean Square") 0 valor de $10^{-6}$. Para confiabilidade dos resultados foi feito o estudo da independência das malhas. Este estudo visa determinar a malha a partir da qual refinamentos não alteram significativamente os resultados obtidos. Foram utilizadas curvas de taxa de circulação para as vazões de 100 a $500 \mathrm{NI} / \mathrm{min}$ como critério de escolha da melhor malha. Encontrou-se que a malha com I.000.000 de elementos de volume foi a mais adequada, pois refinamentos posteriores não afetaram a taxa de circulação segundo o critério proposto.

\section{I Condições de Contorno Adotadas no Desgaseificador RH}

As condições no contorno foram estabelecidas de acordo com as condições físicas nos contornos do domínio do $\mathrm{RH}$. Foram consideradas as seguintes condições de contorno do desgaseificador $\mathrm{RH}$ industrial e do seu modelo físico:

- Paredes: condição de não-escorregamento para os líquidos. Para os gases foi considerada a condição de escorregamento livre ("free slip");

- Superfície livre da panela: a superfície livre da panela foi considerada plana e uma condição de abertura foi adotada;

- Superfície Livre ao Vácuo: na superfície livre ao vácuo foi considerada uma condição de contorno de saída denominada de condição de desgaseificação, e para a fase líquida esta superfície foi considerada como uma condição de escorregamento livre;

- Bicos de Injeção de Gás: nos bicos de injeção de gás foi considerada a condição de entrada para os gases. A faixa de vazão mássica de entrada de ar para o modelo físico variou de 50 a $500 \mathrm{NI} / \mathrm{min}$.

\subsection{Taxa de Circulação}

A obtenção da taxa de circulação foi feita através da injeção de solução salina na perna de subida e medida de variação da concentração do sal (condutividade elétrica) na perna de descida. A Figura 2 mostra uma vista esquemática

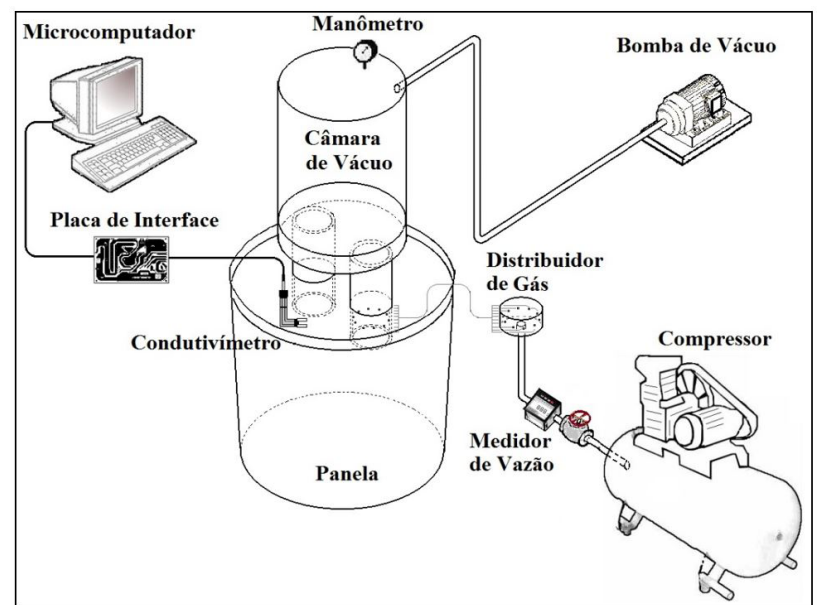

Figura 2. Vista esquemática do modelo físico do reator $\mathrm{RH}$ com os sensores utilizados nos testes.

do aparato experimental com os equipamentos, sensores e placas de aquisição de dados para todos os ensaios.

Para o cálculo da taxa de circulação foi utilizada a técnica adotada por Seshadri e Costa [14]. Aplicando o balanço de massa para o traçador adicionado, encontra-se o tempo necessário para circular um volume de trabalho. Com isso, pode-se determinar a taxa de circulação através da Equação I:

$$
Q=W / \tau
$$

$\tau=$ tempo necessário para circular um volume de trabalho, ou tempo de circulação (s);

$W=$ volume de líquido contido na panela $\left(\mathrm{m}^{3}\right)$.

Foram empregadas vazões de gás, injetado na perna de subida, de 100, 200, 300, 400 e 500 NI/min. Para cada vazão foram realizados testes até obtenção de 10 ensaios válidos com variação máxima de $5 \%$ no fechamento do balanço de massa do traçador.

\section{RESULTADOS E DISCUSSÃO}

\section{I Simulações do Sistema ar/água com o Estudo das Forças de Arraste e de Não-arraste}

Inicialmente foram feitas análises no modelo matemático empregando-se apenas a força de arraste, ou seja, sem forças de não-arraste (SFNA). Estas simulações foram comparadas os resultados experimentais dos ensaios realizados no modelo físico como mostra a Figura 3(a). Nota-se nesta figura que a taxa de circulação calculada pelo modelo matemático aumentou continuamente com o aumento da vazão de gás e que para o resultado experimental a partir da vazão de gás de $300 \mathrm{l} / \mathrm{min}$ a taxa de circulação manteve-se constante. Os resultados de taxa de circulação da simulação matemática utilizando apenas a força de arraste ficaram fora do campo de validação dos resultados obtidos nos ensaios no modelo 

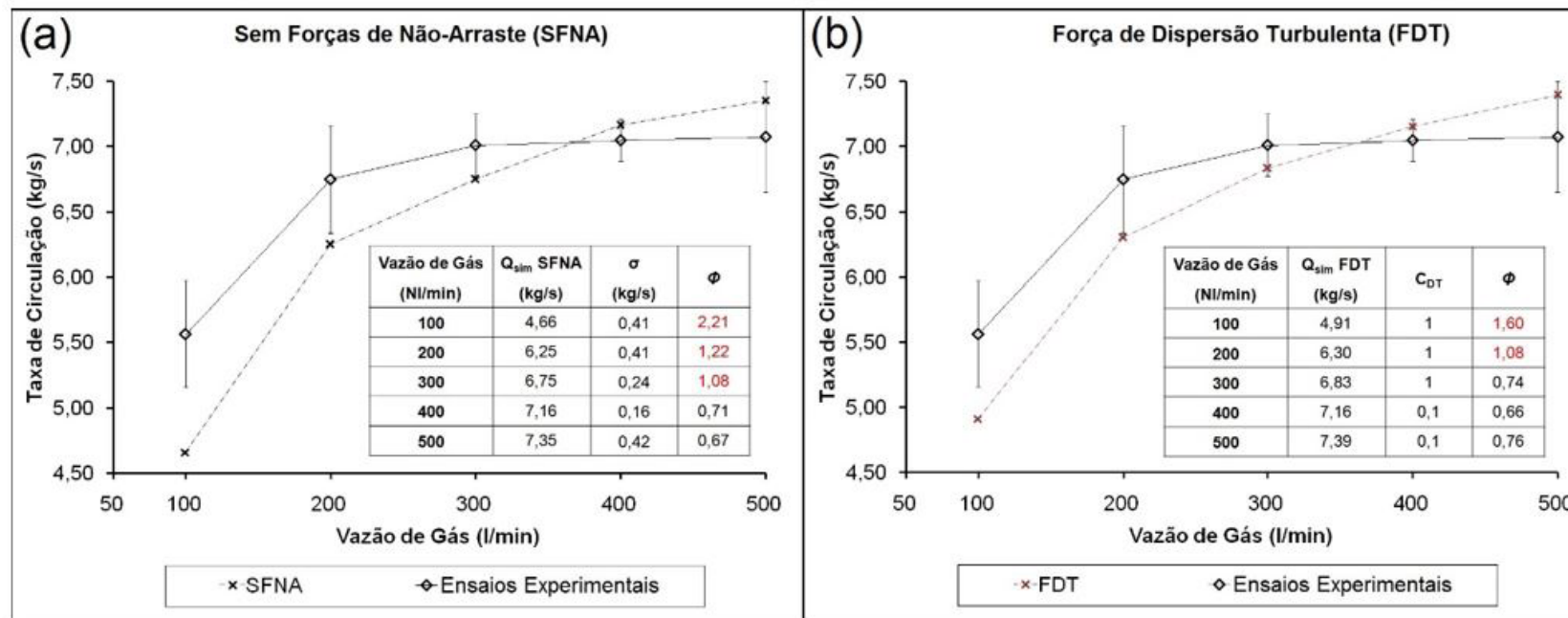

(c)

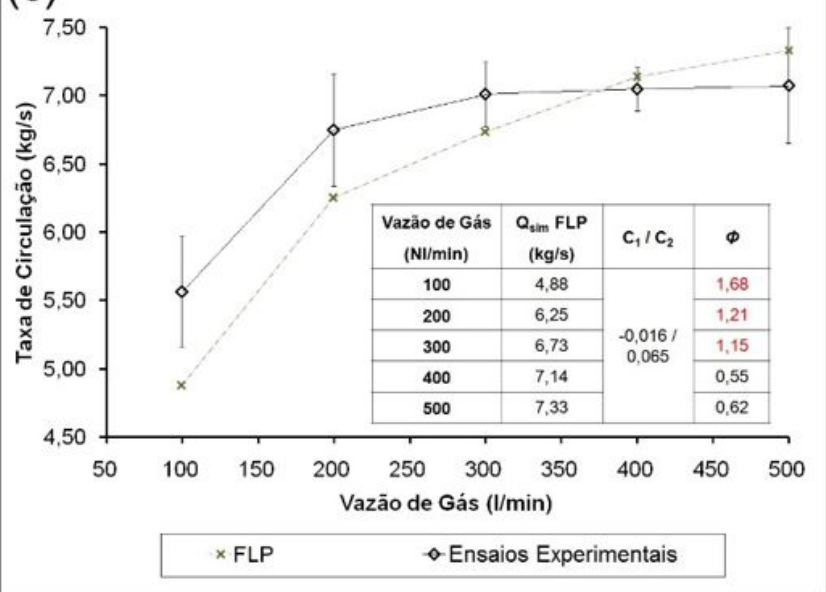

(d)

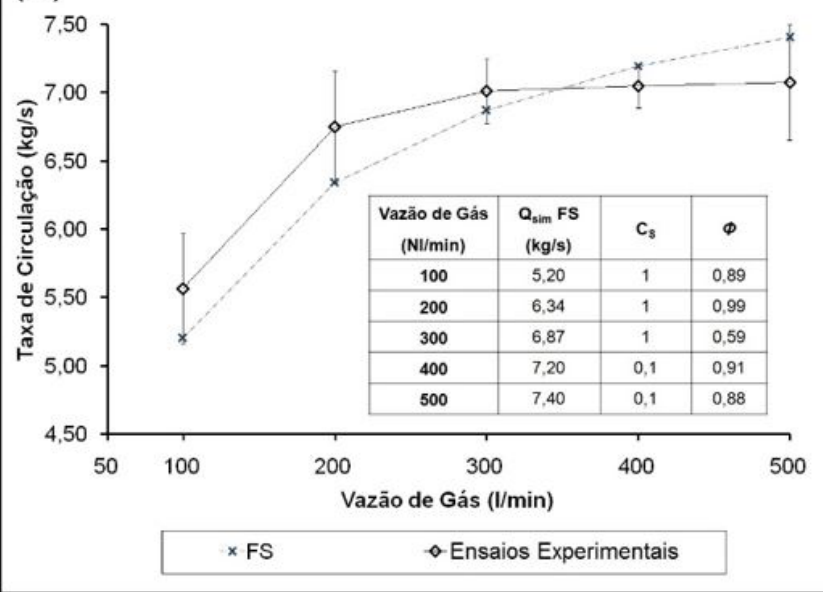

Figura 3. Comparação dos resultados da taxa de circulação versus vazão de gás entre o modelo físico e o matemático nas condições: (a) sem as forças de não-arraste; (b) utilizando a força de lubrificação das paredes; (c) utilizando a força de dispersão turbulenta; (d) utilizando a força de sustentação. Em que SFNA: Sem Forças de Não-Arraste; FLP: Força de Lubrificação das Paredes; FDT: Força de Dispersão Turbulenta; FS: Força de Sustentação e $D_{b}$ : Diâmetros das Bolhas.

físico para as vazões de gás de 100, 200 e 300 l/min. A partir da vazão de $400 \mathrm{l} / \mathrm{min}$ estes valores ficaram dentro dos limites dos resultados experimentais de taxa de circulação.

Uma forma encontrada de avaliar a qualidade das previsões do modelo matemático foi o emprego de um fator representado pela Equação 2:

$$
\varphi=\frac{\left|Q_{\exp }-Q_{\text {sim }}\right|}{\sigma}
$$

sendo

$$
\left\{\begin{array}{cl}
0 \leq \phi \leq 1 & \text { Dentro do Campo de Validação } \\
\phi>1 & \text { Fora do Campo de Validação }
\end{array}\right.
$$

em que:

$\phi=$ fator de validação;

$Q_{\text {exp }}=$ taxa de circulação experimental $(\mathrm{kg} / \mathrm{s})$;

$Q_{\text {sim }}=$ taxa de circulação calculada nas simulações $(\mathrm{kg} / \mathrm{s})$; $\sigma=$ desvio padrão dos resultados experimentais $(\mathrm{kg} / \mathrm{s})$.

O fator de validação não possui valores negativos sendo que o valor de maior precisão é o zero, significando que a previsão do modelo matemático é igual ao valor da média de dez resultados experimentais obtidos nos ensaios. Os resultados da simulação são válidos quando o fator de validação estiverem entre zero e um (valores de taxa de circulação dentro do desvio padrão experimental). Os resultados da simulação não válidos possuem valores acima de um (valores de taxa de circulação acima ou abaixo do desvio padrão experimental). Como o resultado da diferença entre as taxas de circulação do resultado experimental e a previsão obtida na simulação matemática é calculada em valor absoluto, não há valores menores do que zero.

Desta forma, nota-se na Figura 3(a) que apenas as taxas de circulação para as vazões de gás de 400 e $500 \mathrm{l} / \mathrm{min}$ foram validadas, pois possuem o fator de validação dentro do domínio $0 \leq \Phi \leq 1$. 
A Figura 3(b) apresenta os resultados das simulações utilizando a força de dispersão turbulenta comparados com os resultados experimentais para as vazões de 100 a $500 \mathrm{l} / \mathrm{min}$. Os resultados apresentados neste gráfico foram os melhores com o estudo da variação dos coeficientes adimensionais da força de dispersão turbulenta. O melhor valor de coeficiente da força de não-arraste para a vazão de 100,200 e $300 \mathrm{l} / \mathrm{min}$ foi I. Para as vazões de gás de 100 e 200 os resultados ficaram fora do campo de validação. Nota-se que com o emprego da força de dispersão turbulenta, os resultados de taxa de circulação aumentaram para as vazões de 100 e $200 \mathrm{l} / \mathrm{min}$, aproximando-se do campo de validação. Para a vazão de $300 \mathrm{l} / \mathrm{min}$, o resultado foi validado com o coeficiente igual a I. A partir da vazão de gás de $400 \mathrm{l} / \mathrm{min}$ utilizou-se o valor mínimo dos coeficientes adimensionais da força de dispersão turbulenta $(C D T=0, I)$. Os resultados neste trabalho mostram que os coeficientes da força de dispersão turbulenta dependem da vazão de gás utilizada nas simulações e que os melhores resultados foram utilizando os coeficientes igual a I e 0, I, para baixas e altas vazões de gás, respectivamente.

$\mathrm{Na}$ Figura 3(c) foi a analisada a influencia da força de lubrificação das paredes comparados com os resultados experimentais e os melhores resultados são os que utilizaram os coeficientes da força de lubrificação das paredes $C_{1}$ e $C_{2}$ iguais a $-0,016$ e 0,065 .

A análise da força de sustentação e a sua validação são mostrados na Figura 3(d) O melhor resultado obtido foi quando se aplicou o coeficiente da força sustentação igual a I (um) para as vazões de 100 a $300 \mathrm{l} / \mathrm{min}$. Para as vazões de gás 400 e $500 \mathrm{l} / \mathrm{min}$, o coeficiente adimensional foi de $0, I$. Todos os resultados nestas condições foram validados. Há uma maior distribuição da fase dispersa na perna de subida. A força de sustentação ocorre devido ao gradiente de velocidade que ocorre perpendicularmente ao movimento relativo das fases. De acordo com Lucas et al. [15], quando se aumenta o coeficiente de sustentação mantendo seu valor positivo, há a tendência de ocorrer a diminuição da velocidade superficial do líquido na direção do escoamento. No entanto, isto não foi observado neste estudo, pois à medida que se aumentou o coeficiente da força de sustentação, a taxa de circulação aumentou e consequentemente a velocidade da água também aumentou.

Ao se utilizar as forças de não-arraste combinadas encontrou-se resultados dentro e fora do campo de validação para todas as vazões de gás. A Figura 4 mostra a comparação dos resultados de taxa de circulação dos ensaios experimentais com os da simulação matemática com a utilização das forças de dispersão turbulenta, lubrificação as paredes e sustentação combinadas. Vários resultados de taxa de circulação e seus respectivos fatores de validação foram obtidos. Os melhores resultados encontrados foram com os coeficientes das forças de sustentação e lubrificação das paredes constantes enquanto que $\circ$ da força de dispersão turbulenta variou. $O$ coeficiente da força de sustentação $C_{s}$ foi de $0, I$ e os coeficientes da força de lubrificação $C_{1}$ e $C_{2}$ foram de $-0,010$ e 0,050 , respectivamente.
O coeficiente da força de dispersão turbulenta variou de acordo com a vazão de gás, sendo o coeficiente para as vazões de 100 e $200 \mathrm{l} / \mathrm{min}$ foi de $0, \mathrm{I}$, para a vazão de $300 \mathrm{l} / \mathrm{min}$ foi de 0,5 e para as vazóes de 400 e $500 \mathrm{l} / \mathrm{min}$ foi de I; ou seja, baixo para baixas vazões, médio para vazão média e alto para altas vazões. Os valores do fator de validação para as vazões de 100, 200, 300, 400 e $500 \mathrm{l} / \mathrm{min}$ são de 0,$98 ; 0,93 ; 0,98 ; 0,63$ e 0,65 , respectivamente.

Para analisar a razão das diferenças constatadas foram feitas imagens dos resultados das simulações a fração volumétrica de ar nas pernas e na câmara de vácuo do modelo físico do desgaseificador $\mathrm{RH}$ paras as vazões de I00, 200, 300, 400 e $500 \mathrm{l} / \mathrm{min}$, comparando as configurações sem a força de não-arraste e a utilização das forças de não-arraste combinadas, como mostra a Figura 5.

Nota-se nas imagens que há uma tendência de ocorrer uma maior concentração de ar próximo às paredes para a configuração SFNA, isso caracterizado pela pouca penetração, sendo que a força de lubrificação das paredes garante um desprendimento desta pluma das paredes, porém de forma pouco evidenciada.

De uma forma geral, houve um leve aumento da penetração e uma maior dispersão das fases gasosa no interior da perna de subida na região de encontro da pluma, este efeito influenciou no arraste do líquido pelas bolhas. $\mathrm{Na}$ divisão entre a perna de subida e a câmara de vácuo a dispersão da pluma se torna praticamente toda homogênea em relação à seção transversal ao escoamento.

Desta forma, pode-se afirmar que a melhora na dispersão das bolhas influenciou na taxa de circulação ajustando o resultado no intervalo do desvio padrão dos resultados obtidos nos ensaios experimentais.

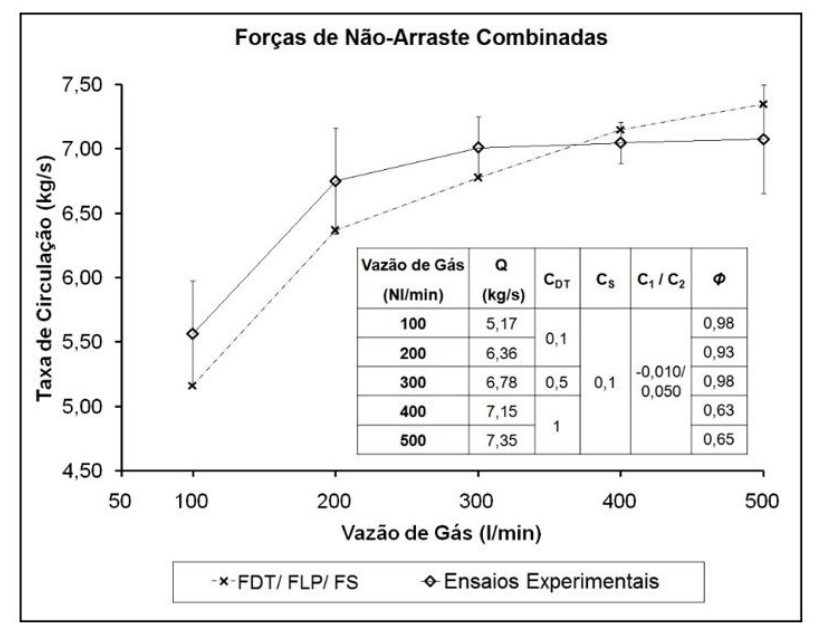

Figura 4. Comparação dos resultados da taxa de circulação versus vazão de gás entre o modelo físico e o matemático nas condições quando se aplica as forças de não-arraste combinadas. FLP: Força de Lubrificação das Paredes; FDT: Força de Dispersão Turbulenta; FS: Força de Sustentação e Db: Diâmetros das Bolhas. 


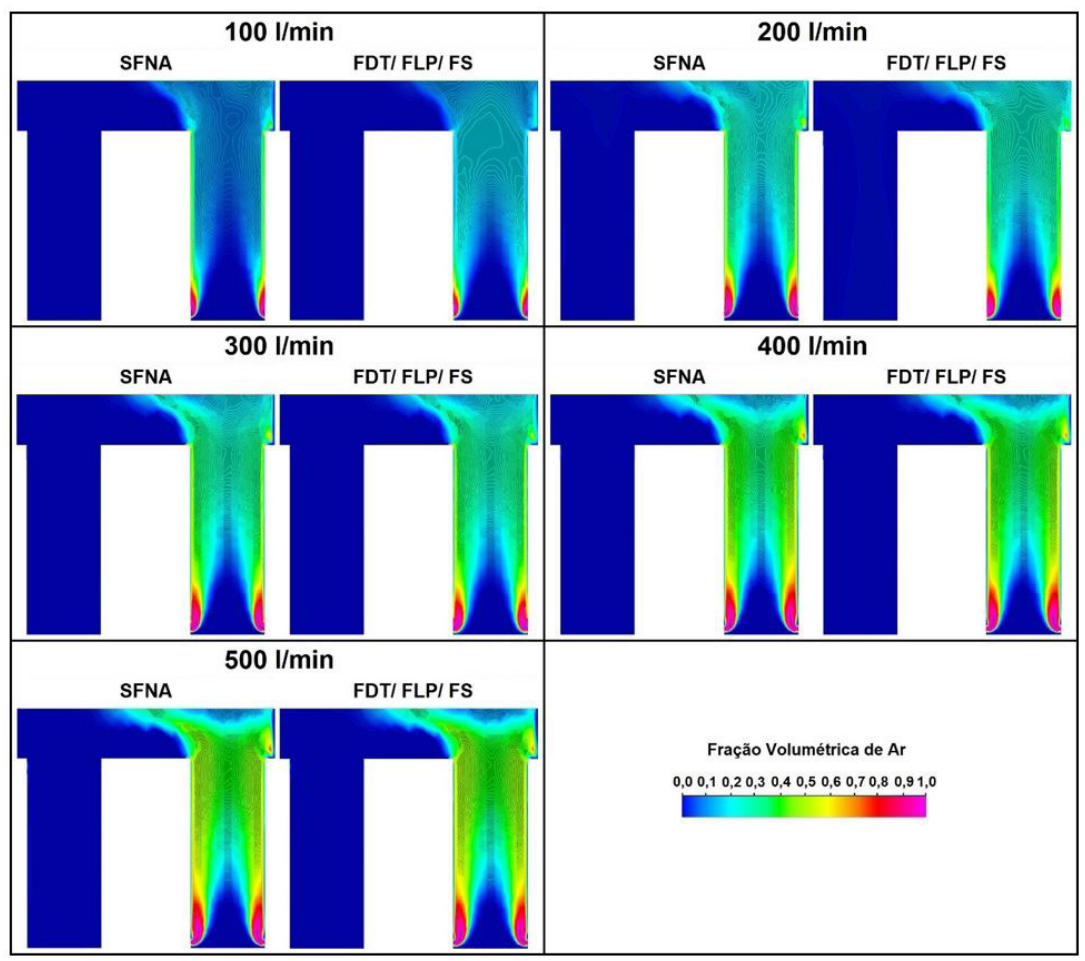

Figura 5. Comparação dos resultados obtidos de fração volumétrica de ar nas pernas e na câmara de vácuo do modelo físico do desgaseificador RH no plano de simetria paras as vazões de 100, 200, 300, 400 e 500 l/min, entre as configurações Sem Força de Não-Arraste (SFNA) e com as forças de não-arraste Força de Dispersão Turbulenta, Força de Lubrificação da Parede e Força de Sustentação combinadas (FDT/FLP/FS).

\section{SUMÁRIO E CONCLUSÕES}

No presente trabalho foi desenvolvido um estudo do escoamento bifásico no modelo físico do desgaseificador $\mathrm{RH}$. Foram feitas simulações matemáticas do desgaseificador $\mathrm{RH}$ considerando sistemas bifásico aço líquido/argônio gasoso. Nos modelos físicos destes reatores, foram considerados sistemas bifásicos ar/água. Para o modelo físico do desgaseificador RH foram realizadas simulações matemáticas considerando diferentes forças de não-arraste. Os resultados das simulações matemáticas foram validados utilizando experimentos em modelos físicos. Para a validação dos resultados da modelagem matemática, ensaios foram feitos utilizando os modelos físicos empregando técnicas para obtenção de tempo de homogeneização de soluções salinas.

As simulações matemáticas do sistema bifásico aplicado ao modelo físico do desgaseificador RH foi feita utilizando as forças de arraste e de não-arraste. As análises mostraram que foi possível validar o modelo matemático utilizando diferentes combinações de força de não-arraste. As análises do efeito das diferentes forças de não arraste foram feitas com a utilização de diferentes coeficientes adimensionais destas forças. As previsões que mais se aproximaram dos resultados experimentais foram utilizando as força de não-arraste combinadas. Sendo o coeficiente da força de sustentação $C_{s}$ de $0, I$ e os coeficientes da força de lubrificação $C_{1}$ e $C_{2}$ de $-0,010$ e 0,050 , respectivamente. $O$ coeficiente da força de dispersão turbulenta variou de acordo com a vazão de gás, sendo o coeficiente para as vazões de 100 e $200 \mathrm{l} / \mathrm{min}$ foi de $0, \mathrm{I}$, para a vazão de $300 \mathrm{l} / \mathrm{min}$ de 0,5 e para as vazões de 400 e $500 \mathrm{l} / \mathrm{min}$ foi de I. Para estes resultados, notou-se que ocorreu uma leve penetração e uma maior dispersão da fases gasosa no interior da perna de subida na região de encontro da pluma. Apesar da boa aproximação e da validação do modelo matemático utilizando forças de não-arraste, novas simulações devem ser propostas para um melhor ajuste dos resultados.

\section{REFERÊNCIAS}

I Li B, Tsukihashi F. Effect of rotating magnetic field on two-phase flow in rh vacuum degassing vessel. ISIJ International. 2005;45(7):972-978.

2 Kishan PA, Dash SK. Prediction of circulation rate in the RH degasser using discrete phase particle modeling. ISIJ International. 2009;49(4):495-504. 
3 Geng DQ, Lei H, He JC. Numerical simulation of the multiphase flow in the Rheinsahl-Heraeus (RH) system. Metallurgical and Materials Transactions B. 20I0;4IB:234-247.

4 Geng D-Q, Zheng J-X, Wang K, Wang P, Liang R-Q, Liu H-T, et al. Simulation on decarburization and inclusion removal process in the Ruhrstahl-Heraeus (RH). Process with Ladle Bottom Blowing Metallurgical and Materials Transactions B. 2015;46(3): |484- 1493.

5 Chen G, He S. Mixing behavior in the RH degasser with bottom gas injection. Vacuum. 20 I6; I 30:48-55.

6 Lopez De Bertodano M, Moraga FJ, Lahey RT Jr. The Modeling of Lift and Dispersion Forces in Two-Fluid Model Simulations of a Bubbly Jet. Journal of Fluids Engineering. 2004; I26(4):573-577.

7 Buwa W, Deo DS, Ranade W. Eulerian-lagrangean simulations of unsteady gas-liquid flows in bubble columns. International Journal of Multiphase Flow. 2006;32(7):864-885.

8 Krepper E, Koncar B, Egorov Y. CFD modeling of subcooled boiling - concept, validation and application to fuel assembly design. Nuclear Engineering and Design. 2007;237:716-73I.

9 Silva CA, Silva IA, Martins EMC, Seshadri V, Perim CA, Vargas GA Fo. Fluid flow and mixing characteristics in RH degasser of Companhia Siderúrgica de Tubarão, and influence of bottom gas injection and nozzle blockage through physical modelling study. Ironmaking \& Steelmaking. 2004;3 I (I):37-42.

I0 Almeida ATP, Alvarenga JA, Belarmino TJP, Cardoso ACP, Nascimento AA, Tavares RP. Physical modeling of vacuum decarburization in an RH degasser. In: Association for Iron \& Steel Technology. Proceedings of the AISTech 2006; 2006 May I-4; Cleveland, USA. Cleveland: AISTech; 2006. p. 76I-770.

I I Lopez De Bertodano M. Two fluid model for two-phase turbulent jet. Nuclear Engineering and Design. 1998; | 79:65-74.

I 2 Grace JR, Wairegi T, Nguyen TH. Shapes and velocities of single drops and bubbles moving freely through immiscible liquids. Transactions of the Institution of Chemical Engineers. 1976;54: I67-I73.

I3 ANSYS $^{\circledR}$ CFX ${ }^{\mathrm{TM}}$. Library materials: pre user's guide. Release II.0. Canonsburg: ANSYS, Inc., 2017.

I4 Seshadri V, Costa SLS. Cold Model of RH Degassing. Transactions of ISIJ. I 986; 26: I 33-I 38.

I5 Lucas D, Shi J-M, Krepper E, Prasser H-M. Models for the forces acting on bubbles in comparison with experimental data for vertical pipe flow. In: 3rd International Symposium on Two-Phase Flow Modeling and Experimentation; 2004 September 22-25; Pisa, Italy. Rome: ENEA; 2004. p. 22-24.

Recebido em: 4 Maio 2016

Aceito em: 9 Fev. 2017 